\title{
A randomized first-passage problem for drifted Brownian motion subject to hold and jump from a boundary
}

\author{
Mario Abundo*
}

\begin{abstract}
We study an inverse first-passage-time problem for Wiener process $X(t)$ subject to hold and jump from a boundary $c$. Let be given a threshold $S>X(0) \geq c$, and a distribution function $F$ on $[0,+\infty)$. The problem consists in finding the distribution of the holding time at $c$ and the distribution of jumps from $c$, so that the first-passage time of $X(t)$ through $S$ has distribution $F$.
\end{abstract}

Keywords: First-passage time, one-dimensional diffusion, random jump, holding time. Mathematics Subject Classification: 60J60,60H05, 60H10.

\section{Introduction}

In this brief note, we generalize the inverse first-passage-time problem for Wiener process $X$ with random jumps from a boundary $c$, studied in our paper [2], by introducing holding when $X$ hits $c$. Diffusions with holding and jumping boundary (DHJ) were first introduced by Feller ([12]), and their ergodic properties were studied in [19], and in [9] in the case without holding. In the one dimensional case, a DHJ coincides with a diffusion in $I=(c, d)$, until it hits the boundary $c$; when this occurs, the process waits there for an independent random duration following an exponential distribution with parameter $\beta>0$, and then it makes a random jump in the interior of $I$, according to a given distribution $\nu$, and starts the diffusion afresh. The (direct) first-passage time (FPT) of a one dimensional DHJ through a barrier $S$ with $c \leq X(0)<S \leq d$, was investigated in [18], while in [5] the FPT was studied in the case without holding, i.e. $\beta=+\infty$ (see also [20]).

The drifted Brownian motion with holding and jump boundary $c$ can be described as follows. Let $\widetilde{X}_{\mu}(t)=x+\mu t+B_{t}$, where $B$ is standard Brownian motion (BM), and $x \in$ $I=[c, d]$. We construct a new stochastic process $X$, called drifted Brownian motion with holding and jump boundary $c$ : starting from $x \in I$, at time $t=0$, we set $X(t)=\widetilde{X}_{\mu}(t)$ until the (random) time at which the boundary $c$ is reached, then the process $\widetilde{X}_{\mu}$ is killed and it is continued as the new process $X$ which holds for an independent exponential time at $c$, with the holding rate $\beta$, then it makes a random jump from $c$ inside $I$ and it starts afresh (independently of the past history) from a point $U \in I$ which is a random variable

*Dipartimento di Matematica, Università "Tor Vergata", via della Ricerca Scientifica, I-00133 Rome, Italy. E-mail: abundo@mat.uniroma2.it 
with distribution $\nu$, and then it evolves as $\widetilde{X}_{\mu}$, until the boundary $c$ is reached again, and so on. Indeed, in the present paper we take $c=0$ and $d>0$, for the sake of simplicity.

Hitting the boundary $c=0$ by the process $X$ can be interpreted as the occurrence of a catastrophe with delayed effect; after holding, it consists in resetting the state of $X$ to a new value, and this has various applications in the scope of diffusion models in Queueing theory (see e.g. [11]), as well as in Mathematical Finance and in Biology (see [5]). As an example, $X(t)$ can represent the number of individuals of a population at time $t$ (for instance fishes in a little lake); whenever the population goes extinct (i.e. it reaches the level 0), after a waiting time, it is performed a restocking by a random amount $U>0$.

Notice that, considering $\widetilde{X}_{\mu}(0) \geq c$ and $c=0$, is not restrictive. For instance, let us consider the diffusion $Z$ with holding and jumping boundary $c \geq Z(0)$, associated to $\widetilde{Z}(t)=$ $\widetilde{Z}(0)+\mu t+B_{t}$ with $\mu>0, Z(0)>0$, and $U \in(0, c) . Z$ is similar to the Wiener-type neuronal model in the presence of refractoriness, considered in [8], [18, for which the neuron fires when its voltage exceeds the threshold $c$, and after the refractoriness period, the voltage is reset to $U \in(0, c)$. We observe that the process $Z$ can be reduced to our case; indeed, by using that $-B_{t}$ is distributed as $B_{t}$, it follows that the distribution of the first hitting time

of $\widetilde{Z}$ to $c$, when starting from $\widetilde{Z}(0) \leq c$, is nothing but the distribution of the first hitting time of $\widetilde{X}_{-\mu}$ to zero, when starting from $c-\widetilde{Z}(0) \geq 0$.

Let $S \in I=(0, d)$ be a constant barrier, and suppose that the holding rate $\beta$ and the jump distribution $\nu(u)=P(U \leq u), u \in(0, S)$ are assigned; then, the FPT of $X$ over $S$, when starting from $0<x<S$, is:

$$
\tau_{S}(x)=\tau_{S, \beta, \nu}(x)=\inf \{t>0: X(t)>S \mid X(0)=\tilde{X}(0)=x\} .
$$

The direct FPT problem for $X$ was studied in [18], while the FPT for the process without holding (i.e. $\beta=+\infty$ ) was studied in [5]; here, we deal with the following inverse FPT (IFPT) problem:

for a given barrier $S \in I$ and $0<x<S$, let $F$ be a distribution on $[0,+\infty)$,

then the IFPT problem consists in finding the holding rate $\beta$ and the jump distribution $\nu$ or its density $g$, if it exists, so that $\tau_{S}(x)$ has distribution $F$.

In this case, we will say that the the pair $(g, \beta)$ is solution to the IFPT problem; notice that the density $g$ has support $(0, S)$.

This IFPT problem is analogous to that considered in 2] for diffusion with only jumps (without holding) from a boundary, and studied in [3], 4], [13] for diffusions without holding and without jumps, and in [1] for reflected diffusions. It has interesting applications in Mathematical Finance, in particular in credit risk modeling, where the FPT represents a default event of an obligor (see [13]), and in Biology (see [17]). The paper is organized as follows: Section 2 contains the main results; Section 3 provides some extension to more general diffusions and in Section 4 we report some explicit examples.

\section{Main results}

Let $\widetilde{X}$ be a one-dimensional diffusion on $D=(0,+\infty)$ and let $X$ the associated diffusion with holding and jumps from the boundary $c=0$, starting from $x>0$. Following the notations of [18], we denote by $\beta>0$ the holding rate of $X$ at 0 , and by $\nu$ the jump distribution 
when it hits 0 . Moreover, let $\tau(x)$ be the first-passage time (FPT) of $X$ through $S$, with $x<S$, and let $h_{S}(t, x)$ the density of $\tau(x)$ and $\widehat{h}_{S}(\lambda, x)$ its Laplace transform $(\lambda>0)$. Finally, denote by $G_{\lambda},(\lambda>0)$ the potential (or resolvent) operator of $X$, which is given by $G_{\lambda} \psi(x)=E^{x} \int_{0}^{+\infty} e^{-\lambda t} \psi(X(t)) d t$, for any Borel function $\psi$ on $[0,+\infty)$, and by $G_{\lambda}^{D}$ the potential operator of $\widetilde{X}$ on $D$, and killed at $\tau_{D}(x)$, i.e. the hitting time of $\widetilde{X}$ to 0 . We recall the following result from [18].

Theorem 2.1 Let $p^{D}$ denote the transition density of the diffusion $\widetilde{X}$ killed at zero; then, the Laplace transform of the FPT of the DHJ $X$ through $S$ is:

$$
\widehat{h}_{S}(\lambda, x)=\frac{\widehat{p}(\lambda, x, S)}{\widehat{p}(\lambda, S, S)}
$$

where $\widehat{p}$ is given by:

$$
\widehat{p}(\lambda, x, y)=\widehat{p}^{D}(\lambda, x, y)+\left(1-\lambda G_{\lambda}^{D} \mathbf{1}(x)\right) \cdot \frac{\delta(y)+\beta \widehat{p}^{D}(\lambda, \nu, y)}{\lambda\left(1+\beta\left\langle\nu, G_{\lambda}^{D} 1\right\rangle\right)} .
$$

Let $\widetilde{X}$ be BM with drift $\mu$, namely, $\widetilde{X}(t)=\widetilde{X}_{\mu}(t)=x+\mu t+B_{t}$, then the transition density of $\widetilde{X}$ killed at 0 is (see [10], pg. 221):

$$
p^{D}(t, x, y)=\frac{1}{\sqrt{2 \pi t}}\left[e^{-(y-\mu t-x)^{2} / 2 t}-e^{-2 \mu x} e^{-(y-\mu t+x)^{2} / 2 t}\right] .
$$

By calculations analogous to those concerning the case $\mu=0$ (see e.g. [21], pg. 82, [14], pg. 354, [15], pg. 288) we obtain its Laplace transform:

$$
\widehat{p}^{D}(\lambda, x, y)=\frac{e^{\mu(y-x)}}{\sqrt{2 \lambda+\mu^{2}}}\left[e^{-|y-x| \sqrt{2 \lambda+\mu^{2}}}-e^{-|y+x| \sqrt{2 \lambda+\mu^{2}}}\right] .
$$

Thus, if $\nu(d x)=g(x) d x$, by straightforward calculations and using the expression of the Laplace transform of the first hitting time of drifted BM to the barrier $S$ (see [15]), (2.2) becomes:

$$
\widehat{p}(\lambda, x, y)=\widehat{p}^{D}(\lambda, x, y)+e^{-x\left(\sqrt{\mu^{2}+2 \lambda}+\mu\right)} \cdot \frac{\delta(y)+\beta \int_{0}^{\infty} \widehat{p}^{D}(\lambda, x, y) g(x) d x}{\lambda+\beta\left(1-\int_{0}^{\infty} e^{-x\left(\sqrt{\mu^{2}+2 \lambda}+\mu\right)} g(x) d x\right)}
$$

where $\widehat{p}^{D}$ is given by (2.4).

Our main result is the following, where, for the sake of simplicity we limit ourselves to consider the IFPT problem for the DHJ $X$ associated to BM with drift $\mu \leq 0$, in the special case when the starting point is $x=0$.

Theorem 2.2 Let $\widetilde{X}(t)=\mu t+B_{t}, \mu \leq 0$, and let us consider the associated process $X$ with holding and jumps from 0 , having holding rate $\beta>0$ and jump distribution $\nu$; suppose that the FPT of $X$ over $S>0$ has an assigned probability density $f(t)=f_{\mu}(t)$ and denote by $\widehat{f}_{\mu}(\lambda)=\int_{0}^{\infty} e^{-\lambda t} f_{\mu}(t) d t, \lambda \geq 0$, the Laplace transform of $f_{\mu}$. Then, if there exists a solution 
$(g, \beta)$ to the IFPT problem for $X$, the Laplace transform $\widehat{g}(\lambda)$ of $g$, and $\beta$ must satisfy the equation:

$$
\begin{aligned}
& \widehat{f}_{\mu}(\lambda)\left[\left(1-e^{-2 S \sqrt{2 \lambda+\mu^{2}}}\right)\left(\lambda+\beta\left(1-\widehat{g}\left(\sqrt{2 \lambda+\mu^{2}}+\mu\right)\right)\right)\right. \\
& \left.+\beta e^{-S\left(2 \sqrt{\mu^{2}+2 \lambda}+\mu\right)}\left(\widehat{g}\left(-\sqrt{2 \lambda+\mu^{2}}\right)-\widehat{g}\left(\sqrt{2 \lambda+\mu^{2}}\right)\right)\right] \\
& =\beta e^{-S\left(\sqrt{2 \lambda+\mu^{2}}-\mu\right)}\left(\widehat{g}\left(-\sqrt{2 \lambda+\mu^{2}}\right)-\widehat{g}\left(\sqrt{2 \lambda+\mu^{2}}\right)\right)
\end{aligned}
$$

Moreover, if $\mu=0$, and we require that the density $g$ is symmetric with respect to $\frac{S}{2}$, then formula (2.6) can be explicited and we get:

$$
\widehat{g}(\lambda)=\frac{\widehat{f_{0}}\left(\frac{\lambda^{2}}{2}\right)\left(\frac{\lambda^{2}}{2}+\beta\left(1+e^{-S \lambda}\right)\right)}{\beta\left(1+\widehat{f}_{0}\left(\frac{\lambda^{2}}{2}\right)\right.} .
$$

or

$$
\widehat{f}_{0}(\lambda)=\frac{\beta \widehat{g}(\sqrt{2 \lambda})}{\lambda+\beta\left(1+e^{-S \sqrt{2 \lambda}}\right)-\beta \widehat{g}(\sqrt{2 \lambda})}
$$

Proof. Setting $\widehat{f}_{\mu}(\lambda)=\widehat{h}_{S}(\lambda, 0)$, by Theorem 2.1, we have

$$
\widehat{f}_{\mu}(\lambda)=\frac{\widehat{p}(\lambda, 0, S)}{\widehat{p}(\lambda, S, S)}
$$

By using (2.5) and calculating the various quantities, it is easy to see that:

$$
\widehat{p}(\lambda, 0, S)=\frac{\beta}{\sqrt{2 \lambda+\mu^{2}}} \cdot \frac{e^{-S\left(\sqrt{2 \lambda+\mu^{2}}-\mu\right)}\left(\widehat{g}\left(-\sqrt{2 \lambda+\mu^{2}}\right)-\widehat{g}\left(\sqrt{2 \lambda+\mu^{2}}\right)\right)}{\lambda+\beta\left(1-\widehat{g}\left(\sqrt{2 \lambda+\mu^{2}}+\mu\right)\right)}
$$

and

$$
\begin{gathered}
\widehat{p}(\lambda, S, S)=\frac{1-e^{-2 S \sqrt{2 \lambda+\mu^{2}}}}{\sqrt{2 \lambda+\mu^{2}}} \\
+\frac{\beta}{\sqrt{2 \lambda+\mu^{2}}} \cdot \frac{e^{-S\left(2 \sqrt{2 \lambda+\mu^{2}}+\mu\right)}\left(\widehat{g}\left(-\sqrt{2 \lambda+\mu^{2}}\right)-\widehat{g}\left(\sqrt{2 \lambda+\mu^{2}}\right)\right)}{\lambda+\beta\left(1-\widehat{g}\left(\sqrt{2 \lambda+\mu^{2}}+\mu\right)\right)}
\end{gathered}
$$

from which (2.6) follows. If $g$ is symmetric with respect to $\frac{S}{2}$, we have:

$$
\widehat{g}(-\lambda)=e^{S \lambda} \widehat{g}(\lambda)
$$

Then, equation (2.7) easily follows, by taking $\mu=0$ in (2.6) and by using (2.10).

Remark 2.3 If there is no holding at zero (i.e. $\beta=+\infty$ ), by letting $\beta \rightarrow+\infty$ in (2.7), we obtain:

$$
\widehat{g}(\lambda)=\frac{\left.\widehat{f}_{0}\left(\frac{\lambda^{2}}{2}\right)\left(1+e^{-S \lambda}\right)\right)}{1+\widehat{f}_{0}\left(\frac{\lambda^{2}}{2}\right)} .
$$

which coincides with equation (2.11) of [2] . 
Remark 2.4 By taking the first and second derivative with respect to $\lambda$ in (2.7) and calculating them at $\lambda=0$, we get

$$
E\left(\tau_{S}(0)\right)=\frac{1}{\beta}+1-2 E\left(U^{2}\right)
$$

For $\beta \rightarrow+\infty$, the above equation yields $E\left(\tau_{S}(0)\right)=1-2 E\left(U^{2}\right)$ (cf. Remark 2.4 of [2]).

Remark 2.5 A result analogous to that of Theorem 2.2 can be also obtained for $x \neq 0$; however, the involved calculations are very heavy and cumbersome, so we have chosen not to develop them.

Remark 2.6 Once the pair $(\widehat{g}, \beta)$ has been found, such that it verifies (2.6), or (2.7), it may be that $\widehat{g}$ is not the Laplace transform of the density function of a random variable $U$ with support $(0, S)$. In this case, a solution to the IFPT problem does not exist. This is the reason why Theorem 2.2 is formulated in a conditional form. This kind of difficulty in showing the existence of a solution to an inverse FPT problem is common to other types of inverse problem (see e.g. [1], [2], [3], [4], [6], [22]); as far as the present IFPT problem is concerned, the difficulties are far stronger, because the relation between $\widehat{g}$ and $\widehat{f}$ is more complicated. For instance, if $\mu=0$ and $f$ is the exponential density with parameter 1 , then the solution to the IFPT problem with $S=1$ and $g$ symmetric in $(0,1)$ does not exist. In fact, suppose that the solution exists, then by (2.7) it follows that

$$
\widehat{g}(\lambda)=\frac{\lambda^{2}+2 \beta\left(1+e^{-\lambda}\right)}{\beta\left(4+\lambda^{2}\right)},
$$

which is not the Laplace transform of a probability density in $(0,1)$, since the third moment is negative.

Taking into account Remark [2.6, we will prove the existence of the density $g$ of $U$ for a class of FPT densities $f$. For the sake of simplicity, we limit ourselves to the case when $\mu=0, x=0, S=1$, and $g$ is required to be a function with support in $(0,1)$, which is symmetric with respect to the middle point $1 / 2$; in fact, for $\mu \neq 0$ the calculations involved are far more complicated.

For an integer $k \geq 0$, set $I_{k}(\lambda)=\int_{-1}^{1} e^{-\lambda x} x^{k} d x$; as easily seen, $I_{0}(\lambda)=2 \sinh (\lambda) / \lambda$ and the recursive relation $I_{k}(\lambda)=\frac{(-1)^{k} e^{\lambda}-e^{-\lambda}}{\lambda}+\frac{k}{\lambda} I_{k-1}(\lambda)$ allows to calculate $I_{k}(\lambda)$, for every $k$.

The following Proposition gives a sufficient condition, so that there exists the solution $(g, \beta)$ to the IFPT problem for the process $X$ with holding and jumps from zero, associated to $\widetilde{X}(t)=B_{t}$, and the barrier $S=1$.

Proposition 2.7 Let $\widetilde{X}(t)=B_{t}$, and suppose that the Laplace transform of $f(t)$ has the form:

$$
\widehat{f}(\lambda)=\widehat{f}_{2 k}(\lambda)=\frac{\left(1+\frac{1}{2 k}\right) e^{-\sqrt{\lambda / 2}}\left[\sqrt{2 / \lambda} \sinh (\sqrt{\lambda / 2})-I_{2 k}(\sqrt{\lambda / 2})\right]}{1+e^{-\sqrt{2 \lambda}}+\lambda / b-\left(1+\frac{1}{2 k}\right) e^{-\sqrt{\lambda / 2}}\left[\sqrt{2 / \lambda} \sinh (\sqrt{\lambda / 2})-I_{2 k}(\sqrt{\lambda / 2})\right]},
$$

for $b>0$ and some integer $k>0$. Then, there exists the solution $(g, \beta)$ of the IFPT problem for $X$, relative to the barrier $S=1$ and the FPT density $f$, and it results $\beta=b$ and:

$$
g(u)=g_{2 k}(u)=\left(1+\frac{1}{2 k}\right)\left(1-(2 u-1)^{2 k}\right), k \geq 0, u \in(0,1) .
$$


Proof. A simple calculation shows that

$$
\widehat{g}_{2 k}(\lambda)=\left(1+\frac{1}{2 k}\right) e^{-\lambda / 2}\left[\frac{2}{\lambda} \sinh (\lambda / 2)-I_{2 k}(\lambda / 2)\right] .
$$

Since $g_{2 k}$ is symmetric with respect to $S / 2=1 / 2$, the result follows by inserting $\widehat{g}_{2 k}$ into (2.7).

Notice that, letting $b$ go to $+\infty$, (2.13) becomes equation $(2.17)$ of [2].

Remark 2.8 A straightforward calculation shows that, if $U \in(0,1)$ has density $g_{2 k}$, then $E\left(U^{2}\right)=\frac{4 k+5}{6(2 k+3)}$. Then, by using (2.12), we obtain that the FPT-distribution corresponding to $\widehat{f}_{2 k}$ has mean $E\left(\tau_{1}(0)\right)=\frac{2(k+2)}{3(2 k+3)}+\frac{1}{b}$.

\section{Diffusions conjugated to Brownian motion}

In certain cases, a one-dimensional diffusion $\widetilde{X}$ can be reduced to BM by a variable change; by using this approach, we shall extend to a general one-dimensional DHJ $X$, which is associated to $\widetilde{X}$, the results obtained for Wiener process. For $a>0$, let $J=[0, a]$ or $J=[0, a)$, with $a \leq+\infty$, and suppose that $\tilde{X}$ is a time-homogeneous diffusion in $J$ which is the solution of the stochastic differential equation (SDE):

$$
d \tilde{X}(t)=\mu(\tilde{X}(t)) d t+\sigma(\tilde{X}(t)) d B_{t}, \tilde{X}(0)=x \in J
$$

where the coefficients $\mu(x)$ and $\sigma(x)$ are regular enough functions (see e.g. [2]), so that a unique strong solution exists. We consider the following:

Definition 3.1 We say that $\widetilde{X}$ is conjugated to $B M$ if there exists an increasing function $v: J \longrightarrow \mathbb{R}$ with $v(0)=0$, such that:

(i) $v(x)$ is continuous for any $x \in J$ and it is differentiable in the interior of $J$;

(ii) $v^{-1}(y)$ is differentiable in the interior of $v(J)$ and it possesses the right derivative at

$y=0$ and the left derivative at $y=v(a)$, if $a<+\infty$.

(iii) $\tilde{X}(t)=v^{-1}\left(B_{t}+v(x)\right)$, for any $t \geq 0$.

Let us suppose that $\widetilde{X}$ is conjugated to BM, and consider the associated process $X$ with holding and jumps from 0 . Notice that holding and random reflection of $\widetilde{X}$ at zero corresponds to holding and random reflection of $B_{t}+v(x)$ at zero; moreover the first passage $\widetilde{\tau}_{S}(x)$ of $\widetilde{X}$ through the barrier $S$, with $0<S<a$, corresponds to the first passage of $B_{t}+v(x)$ through $v(S)$, and $\widetilde{\tau}_{S}(x)=\widetilde{\tau}_{v(S)}^{B}(v(x))$, where the superscript $B$ refers to BM. Furthermore, let $g(u)$ be the density of the position $U \in(0, S)$ from which, once $X$ has hit 0 , it starts afresh, after the holding time, and let $q(y)$ be the corresponding density of the position $V=v(U) \in(0, v(S))$ from which, once $B_{t}+v(x)$ has hit 0 , it starts afresh, after the holding time; then, $q(y)=g\left(v^{-1}(y)\right)\left(v^{-1}\right)^{\prime}(y), y \in(0, v(S))$. Thus, if $\widetilde{X}$ is conjugated to BM via the function $v$, then the solution $(g, \beta)$ to the IFPT problem for the process $X$ associated to $\tilde{X}$, relative to the FPT density $f$ and the barrier $S$, can be written in terms of the solution $(q, \beta)$ to the IFPT problem for the process associated to $v(x)+B_{t}$, relative to the FPT density $f$ and the barrier $v(S)$, by using that $g(x)=q(v(x)) v^{\prime}(x)$. As easily seen, if $x=0, \widehat{q}$ is obtained by (2.6) with $\mu=0$, with $\widehat{q}$ in place of $\widehat{g}$ and $v(S)$ in place of $S$. 


\section{A few examples}

Example 1 (when $g$ is the uniform density in $(0, S)$ )

Let $\tilde{X}(t)=B_{t}$ and $S>0, b>0$ and let

$$
\widehat{f}(\lambda)=\frac{b\left(1-e^{-S \sqrt{2 \lambda}}\right)}{S \sqrt{2 \lambda}\left[\lambda+b\left(1+e^{-S \sqrt{2 \lambda}}\right)\right]-b\left(1-e^{-S \sqrt{2 \lambda}}\right)} .
$$

Then, the solution to the IFPT problem for $X$ relative to $S$ is the pair $(g, \beta)$ with $\beta=b$ and $g(u)=\frac{1}{S} \mathbf{1}_{(0, S)}(u)$, i.e. the uniform density in $(0, S)$; this is easily obtained by searching for a solution which is symmetric with respect to $S / 2$; by using (2.7) with $\beta=b$, we get $\widehat{g}(\lambda)=\frac{1-e^{-S \lambda}}{S \lambda}$ which is the Laplace transform of $g(u)=\frac{1}{S} \mathbf{1}_{(0, S)}(u)$. In the case $S=1$, we can obtain the same result by letting $k$ go to infinity in $\widehat{f}_{2 k}$ and $g_{2 k}$ of Proposition 2.7, moreover, the mean of the FPT-distribution corresponding to $\widehat{f}$ is $1 / 3+1 / b$, as it also follows by calculating $\lim _{k \rightarrow \infty}\left(\frac{2(k+2)}{3(2 k+3)}+\frac{1}{b}\right)$ (see Remark 2.8 ). For $b \rightarrow+\infty$ (no holding at 0 ), one obtains the function $\widehat{f}(\lambda)$ of Example 3 of [2].

Example 2 Let $\widetilde{X}(t)=B_{t}$ and $S>0, b>0$ and let

$$
\widehat{f}(\lambda)=\frac{b \pi^{2}\left(1+e^{-S \sqrt{2 \lambda}}\right)}{\left(4 \lambda S^{2}+2 \pi^{2}\right)\left(\lambda+b\left(1+e^{-S \sqrt{2 \lambda}}\right)\right)-b \pi^{2}\left(1+e^{-S \sqrt{2 \lambda}}\right)}
$$

then the solution to the IFPT problem for $X$ relative to $S$ is the pair $(g, \beta)$ with $\beta=b$ and $g(u)=\frac{\pi}{2 S} \sin \left(\frac{\pi}{S} u\right), u \in(0, S)$.

In fact, we search for a solution which is symmetric with respect to $S / 2$; by using (2.7) with $\beta=b$, it follows that $\widehat{g}(\lambda)=\frac{\pi^{2}}{2} \frac{\left(1+e^{-\lambda S}\right)}{\lambda^{2} S^{2}+\pi^{2}}$, which is indeed the Laplace transform of the function $g(u)$ above. If there is no holding at 0 (i.e. $b=+\infty), \widehat{f}(\lambda)$ becomes that of Example 1 of [2].

Example 3 (when $g$ is a Beta density) Let $\widetilde{X}(t)=B_{t}$ and $S>0, b>0$ and let

$$
\widehat{f}(\lambda)=\frac{6\left(e^{-S \sqrt{2 \lambda}}(S \sqrt{2 \lambda}+2)+S \sqrt{2 \lambda}-2\right)}{S^{3} \lambda^{3}\left(1+e^{-S \sqrt{2 \lambda}}\right)-6\left(e^{-S \sqrt{2 \lambda}}(S \sqrt{2 \lambda}+2)+S \sqrt{2 \lambda}-2\right)+\lambda / b} .
$$

Then, the solution to the IFPT problem for $X$ relative to $S$ is the pair $(g, \beta)$ with $\beta=b$ and $g(u)=\frac{6}{S^{3}} u(S-u), u \in(0, S)$. In fact, by using (2.7) with $\beta=b$, it follows that $\widehat{g}(\lambda)=$ $\frac{6}{S^{3} \lambda^{3}}\left[e^{-S \lambda}(S \lambda+2)+S \lambda-2\right]$, which is indeed the Laplace transform of $g(u)=\frac{6}{S^{3}} u(S-u)$. Notice that, for $S=1, g$ is the density $g_{2 k}$ of Proposition 2.7, for $k=1$. For $b \rightarrow+\infty$ (no holding at 0 ), one obtains the function $\widehat{f}(\lambda)$ of Example 4 of [2].

Example 4 (when $g$ is the triangular density in $(0,1)$ )

Let $\widetilde{X}(t)=B_{t}$ and $S=1, b>0$ and let

$$
\widehat{f}(\lambda)=\frac{2 b\left(1-e^{\sqrt{\lambda / 2}}\right)^{2}}{\lambda\left[\lambda+b\left(1+e^{-\sqrt{2 \lambda}}\right)\right]-2 b\left(1-e^{-\sqrt{\lambda / 2}}\right)^{2}}
$$


Then, the solution to the IFPT problem for $X$ relative to $S$ is the pair $(g, \beta)$ where $\beta=b$ and $g$ is the triangular density in $(0,1)$ :

$$
g(u)=\left\{\begin{array}{l}
4 u, u \in\left(0, \frac{1}{2}\right] \\
4(1-u), u \in\left(\frac{1}{2}, 1\right)
\end{array}\right.
$$

In fact, by using (2.7) with $\beta=b$, it follows that $\widehat{g}(\lambda)=\frac{4}{\lambda^{2}}\left(1-e^{-\lambda / 2}\right)^{2}$, which is indeed the Laplace transform of the function $g(u)$ above. For $b \rightarrow+\infty$ (no holding at 0 ), one obtains the function $\widehat{f}(\lambda)$ of Example 5 of [2].

\section{Example 5.}

A class of diffusions conjugated to $\mathrm{BM}$ is given by processes $\tilde{X}(t)$ which are solutions of SDEs such as

$$
d \tilde{X}(t)=\frac{1}{2} \sigma(\widetilde{X}(t)) \sigma^{\prime}(\tilde{X}(t)) d t+\sigma(\tilde{X}(t)) d B_{t}, \tilde{X}(0)=x
$$

with $\sigma(\cdot) \geq 0$. Indeed, if the integral $v(z) \doteq \int_{x}^{z} \frac{1}{\sigma(r)} d r$ is convergent for every $z$, by Itô's formula, we obtain that $\tilde{X}(t)=v^{-1}\left(B_{t}+v(x)\right)$. Then, by using the results of Section 2 , examples of solutions to IFPT problems with $x=0$ can be easily derived from Examples 1 to 4 , with regard to the process $X(t)$ with holding and jumps from 0 , associated to $\widetilde{X}$ which is driven by the SDE (4.1), for some choice of $\sigma(\cdot)$; in fact, it suffices to replace $S$ with $v(S)$. For instance, diffusions $\widetilde{X}$ of this kind are the well-known Feller process (also known as the Cox-Ingersoll-Ross (CIR) model), and the Wright \& Fisher-like process (see Examples (i) and (ii) of [2]).

Example 6 (Ornstein-Uhlenbeck process)

Let $\widetilde{X}(t)$ be the solution of the SDE:

$$
d \widetilde{X}(t)=-\mu \widetilde{X}(t) d t+\sigma d B_{t}, \tilde{X}(0)=x,
$$

where $\mu, \sigma$ are positive constants. By using a time-change, the explicit solution assumes the form $\widetilde{X}(t)=e^{-\mu t}(x+B(\rho(t)))$, where $\rho(t)=\frac{\sigma^{2}}{2 \mu}\left(e^{2 \mu t}-1\right)$. If $S(t)$ is a moving barrier, the FPT of $\widetilde{X}(t)$ over $S(t)$ is $\widetilde{\tau}_{S(t)}=\inf \left\{t>0: x+B(\rho(t)) \geq e^{\mu t} S(t)\right\}$ and so $\rho\left(\widetilde{\tau}_{S(t)}\right)=$ $\inf \left\{u>0: x+B_{u} \geq \widetilde{S}(u)\right\}$, where $\widetilde{S}(u)=e^{\mu \rho^{-1}(u)} S\left(\rho^{-1}(u)\right)$. Therefore, if $S(t)=S_{0} e^{-\mu t}$, the IFPT problem for the associated DHJ $X(t)$, and relative to the moving barrier $S(t)$ and the FPT distribution $F$, is reduced to the IFPT problem for DHJ associated to BM, starting from $x$ and relative to the constant barrier $S_{0}$ and the FPT distribution $\widetilde{F}=F \circ \rho^{-1}$. Thus, if $x=0$, explicit examples for the Ornstein-Uhlenbeck process and the exponential barrier $S(t)=S_{0} e^{-\mu t}$, can be easily derived from Examples 1 to 4 .

Example 7 (Geometric Brownian motion)

Let $\widetilde{X}(t)$ be the solution of the SDE:

$$
d \tilde{X}(t)=r \tilde{X}(t) d t+\sigma \widetilde{X}(t) d B_{t}, \tilde{X}(0)=x>0
$$

where $r$ and $\sigma$ are positive constant. This is a well-known equation in the framework of Mathematical Finance, since it describes the time evolution of a stock price $\widetilde{X}$. The explicit solution is $\tilde{X}(t)=x e^{\mu t} e^{\sigma B_{t}}$, where $\mu=r-\sigma^{2} / 2$. Let us consider the moving barrier $S(t)=$ $e^{\sigma S+\mu^{\prime} t}$; then, the IFPT problem for the associated DHJ $X(t)$, relative to $S(t)$ and the FPT distribution $F$, is reduced to the IFPT problem for DHJ associated to BM with drift $\left(\mu-\mu^{\prime}\right) / \sigma$, starting from $\frac{\ln x}{\sigma}$ and relative to the constant boundary $S$, and the same FPT distribution $F$. 


\section{References}

[1] Abundo, M. 2014. One-dimensional reflected diffusions with two boundaries and an inverse first-hitting problem. Stochastic Anal. Appl. 32: 4, 975991, DOI: 10.1080/07362994.2014.959595

[2] Abundo, M. 2013. Solving an inverse first-passage-time problem for Wiener process subject to random jumps from a boundary. Stochastic Anal. Appl. 31: 4, 695-707.

[3] Abundo, M. 2013. The double-barrier inverse first-passage problem for Wiener process with random starting point. Statist. Probab. Lett. 83: 168-176.

[4] Abundo, M. 2012. An inverse first-passage problem for one-dimensional diffusions with random starting point. Statist. Probab. Lett. 82(1): 7-14.

[5] Abundo, M. 2011. First passage problems for one-dimensional diffusions with random jumps from a boundary. Stochastic Anal. Appl. 29(1): 121-145.

[6] Abundo, M. 2006. Limit at zero of the first-passage time density and the inverse problem for one-dimensional diffusions. Stochastic Anal. Appl. 24: 1119-1145.

[7] Abundo, M. 1997. On some properties of one-dimensional diffusion processes on an interval. Prob. Math. Statis. 17(2): 235-268.

[8] Albano, G., Giorno, V., Nobile, A.G., and Ricciardi, L.M. 2007. A Wiener-type neuronal model in the presence of exponential refractoriness. BioSystem 88: 202-215.

[9] Ben-Ari, I., Pinsky, R.G. 2009. Ergodic behavior of diffusions with random jumps from the boundary. Stoch. Processes Appl. 119(3): 864-881.

[10] Cox, D.R. and Miller, H.D., 1965. The Theory of Stochastic Processes. Methuen \& Co LTD, London.

[11] Di Crescenzo, A., Giorno, V., Nobile, A.G. and Ricciardi, L.M. 2003. On the M/M/1 queue with catastrophes and its continuous approximation. Queueing System 43: 329347.

[12] Feller, W. 1954. Diffusion processes in one dimension. Trans. Amer. Math. Soc. 17: $1-31$.

[13] Jackson, K, Kreinin, A, and Zhang, W. 2009. Randomization in the first hitting problem. Statist. Probab. Lett. 79: 2422-2428.

[14] Karlin, S. and Taylor, H.M. 1975. A first course in stochastic processes. Academic Press, New York.

[15] Karlin, S. and Taylor, H.M. 1981. A second course in stochastic processes. Academic Press, New York.

[16] Lanska, V., Lansky, P. and Smiths, C.E. 1994. Synaptic transmission in a diffusion model for neural activity. J. Theor. Biol. 166: 393-406. 
[17] Lansky, P. and Smiths, C.E. 1989. The effect of a random initial value in neural firstpassage-time models. Math. Biosci. 93(2): 191-215.

[18] Peng, J. 2014. A note on the first passage time of diffusions with holding and jumping boujndary. Statist. Probab. Lett. 93: 58-64.

[19] Peng, J. and Li, W. 2013. Diffusions with holding and jumping boundary. Sci.China Math. 1: 161-176.

[20] Peng, J. and Zaiming Liu. 2009. On a class of mathematical ecosystems with random jumps. Statist. Probab. Lett. 79(5): 630-636.

[21] Revuz, D. and Yor, M., 1991. Continous martingales and Brownian motion. SpringerVerlag, Berlin Heidelberg.

[22] Zucca, C. and Sacerdote, L. 2009. On the inverse first-passage-time problem for a Wiener process. Ann. Appl. Probab. 19 (4): 1319-1346. 